\title{
OPTIMAL MEASURES FOR THE FUNDAMENTAL GAP OF SCHRÖDINGER OPERATORS
}

\author{
NiCOLAS VARCHON ${ }^{1}$
}

\begin{abstract}
We study the potential which minimizes the fundamental gap of the Schrödinger operator under the total mass constraint. We consider the relaxed potential and prove a regularity result for the optimal one, we also give a description of it. A consequence of this result is the existence of an optimal potential under $L^{1}$ constraints.
\end{abstract}

Mathematics Subject Classification. 35J10, 49K20, 35J20, 35B20.

Received March 17, 2008. Revised June 24, 2008.

Published online December 19, 2008.

\section{INTRODUCTION}

In quantum mechanics, the fundamental gap is the difference between the first two energy levels of a quantum particle. If the particle is placed in a potential field $V(x)$, the fundamental gap is the difference between the first two eigenvalues of the Schrödinger operator $-\Delta+V(x)$. The point of our interest here is the potential minimizing this difference.

More precisely, we want to minimize the fundamental gap among potentials belonging to the class of positive Borel measures, with a mass constraint. Let $\Omega$ be a smooth bounded domain in $\mathbb{R}^{N}$, we consider the following optimization problem:

$$
\inf _{\mu \in \mathcal{M}_{0}(\Omega)}\left\{\lambda_{2}(\mu)-\lambda_{1}(\mu)+\alpha \mu(\Omega)\right\}
$$

where $\mathcal{M}_{0}(\Omega)$ is the set of Borel measures which are absolutely continuous with respect to capacity of sets, $\alpha$ is a nonnegative parameter and $\lambda_{1}(\mu)$ and $\lambda_{2}(\mu)$ are respectively the first and the second eigenvalues of the system:

$$
\left\{\begin{aligned}
-\Delta u_{k}+\mu u_{k} & =\lambda_{k}(\mu) u_{k} & & \text { in } \Omega, \\
u_{k} & =0 & & \text { on } \partial \Omega .
\end{aligned}\right.
$$

The parameter $\alpha$ is a Lagrange multiplier, it allows to take into account the mass constraint. Without constraint, the minimum is equal to zero and is attained by measures which are infinite on subsets $E$ of $\Omega$ such that $\Omega \backslash E$ is not connected. If $\alpha$ is too large, the mass constraint becomes too strong and the minimum is trivial.

The problem of minimizing the fundamental gap among potentials $V$ of a given $L^{p}$ norm has already a large literature; we refer to [12] for a survey of results. The case of $L^{\infty}$ constraint has been studied by several authors,

\footnotetext{
Keywords and phrases. Schrödinger operator, eigenvalue problems, measure theory, shape optimization.

1 Collège Condorcet de Bresles, Rue du Petit Chantilly, 60510 Bresles, France. nicolas.varchon@ac-amiens.fr
} 
and in particular in [1], where the authors consider potentials in the class $\left\{V \in L^{\infty}(\Omega) ; 0 \leq V \leq M\right\}$ and prove that the minimizer is a bang-bang function of the form $V=M \chi_{\omega}$ where $\omega$ is a subset of $\Omega$. They consider also an $L^{p}$ constraint with $p>\frac{N}{2}$; they obtain then that in the class $\left\{V \in L^{p}(\Omega) ;\|V\|_{L^{p}(\Omega)} \leq M\right\}$ the minimizer is a regular function, satisfying $u_{2}^{2}-u_{1}^{2}=-c|V|^{p-2} V$ in $\Omega$, where $u_{1}$ and $u_{2}$ are the eigenfunctions associated respectively to $\lambda_{1}(V)$ and $\lambda_{2}(V)$. Moreover, in both cases, it appears that the second eigenvalue at the optimum is non degenerate.

In this work, we show at first that the problem (1.1) admits a solution and that the second eigenvalue for the optimal potential is non degenerate, as in the case of $L^{p}$ constraints. We prove then that this solution is absolutely continuous with respect to the Lebesgue measure and we give its characterization. This is the main theorem of this work:

Theorem 1.1. Let $\mu$ be a solution of problem (1.1) with $0<\alpha<|\Omega|^{-1}$. Then $\mu$ is absolutely continuous with respect to the Lebesgue measure and verifies

$$
\mu=\frac{2}{\alpha}\left[\lambda_{1}(\mu) u_{1}^{2}-\lambda_{2}(\mu) u_{2}^{2}+\left|\nabla u_{2}\right|^{2}-\left|\nabla u_{1}\right|^{2}\right] \cdot \mathcal{L}_{\left\{u_{1}^{2}-u_{2}^{2}=\alpha\right\}},
$$

where $u_{1}$ and $u_{2}$ are two normalized eigenfunctions associated respectively to $\lambda_{1}(\mu)$ and $\lambda_{2}(\mu)$.

As an immediate consequence of this result, we get existence of a solution to the minimization problem under a constraint of class $L^{1}$ :

$$
\inf _{\substack{V \in L^{1}(\Omega) \\ V \geq 0}}\left\{\lambda_{2}(V)-\lambda_{1}(V)+\alpha\|V\|_{L^{1}(\Omega)}\right\} .
$$

In a sense, we recover the result of [1] for constraints of $L^{p}$ type, the equality $u_{1}^{2}-u_{2}^{2}=\alpha$ on the optimal measure's support corresponding to $u_{2}^{2}-u_{1}^{2}=-c|V|^{p-2} V$ for $p=1$.

The paper is organized as follows: in Section 2, we introduce the mathematical tools. In Section 3, we look for directionnal derivative of eigenvalue and we prove also that for $\alpha$ small enough, the minimum is not attained by the measure identically equal to zero. In Section 4, we show existence of a minimum, give some necessary conditions of optimality and the main Theorem 1.1. In the Appendix, we give the proofs of some technical results dealing with eigenvalues and eigenvectors of the Schrödinger operator.

\section{The mathematical SETting}

Let us now specify the optimization problem we study and the notation used throughout this paper.

In all what follows, $D$ is a bounded open set in $\mathbb{R}^{N}$ and $\Omega$ is a connected smooth open set included in $D$.

The capacity of a subset $A \subset D$ is defined by

$$
\operatorname{Cap}(A)=\inf \left\{\int_{D}|\nabla u|^{2} \mathrm{~d} x: u \in \mathcal{U}_{A}\right\}
$$

where $\mathcal{U}_{A}$ is the set of all functions $u$ of the Sobolev space $H_{0}^{1}(D)$ such that $u \geq 1$ almost everywhere in a neighborhood of $A$.

If a property $P(x)$ holds for all $x \in E$ except for the elements of a set $Z \subset E$ with $\operatorname{Cap}(Z)=0$, we say that $P(x)$ holds quasi-everywhere on $E$ (shortly q.e. on $E$ ). The expression almost everywhere (shortly a.e.) refers, as usual, to the Lebesgue measure.

We denote by $\mathcal{L}$ the $N$-dimensional Lebesgue measure. We denote the average of $f$ over the set $E$ with respect to $\mathcal{L}$ by

$$
\oint_{E} f \mathrm{~d} x=\frac{1}{\mathcal{L}(E)} \int_{E} f \mathrm{~d} x
$$


We recall the classical result about Lebesgue points: let $f$ be a function in $L^{p}\left(\mathbb{R}^{N}\right)$ with $1 \leq p<\infty$ and let $B(x, \varepsilon)$ be the ball of center $x$ and radius $\varepsilon$, then for a.e. $x$ in $\Omega$,

$$
\lim _{\varepsilon \rightarrow 0} \oint_{B(x, \varepsilon)}|f-f(x)|^{p} \mathrm{~d} x=0 .
$$

A point $x$ for which (2.1) holds is called a Lebesgue point.

A function $f: \Omega \mapsto \mathbb{R}$ is said to be quasi-continuous if for every $\varepsilon>0$ there exists a continuous function $f_{\varepsilon}: \Omega \mapsto \mathbb{R}$ such that $\operatorname{Cap}\left(\left\{f_{\varepsilon} \neq f\right\}\right)<\varepsilon$. It is well known that every function $u \in H^{1}\left(\mathbb{R}^{N}\right)$ has a quasicontinuous representative, which is uniquely defined up to a set of capacity zero.

We define the admissible set $\mathcal{M}_{0}(\Omega)$ as the class of all nonnegative Borel measures on $\Omega$ such that $\mu(B)=0$ for every Borel subset $B$ of $\Omega$ for which $\operatorname{Cap}(B)=0$. The expression

$$
\left(\int_{\Omega}|\nabla u|^{2} \mathrm{~d} x+\int_{\Omega} u^{2} \mathrm{~d} \mu\right)^{1 / 2}
$$

defines a norm on the Hilbert space $X_{\mu}=H_{0}^{1}(\Omega) \cap L^{2}(\Omega, \mu)$. Let $f$ be a function in $L^{2}(\Omega)$; when we call $u$ a solution of the Dirichlet problem

$$
-\Delta u+u \mu=f
$$

we mean that $u \in X_{\mu}$ and is the unique solution of the variational problem

$$
\int_{\Omega} \nabla u \nabla v \mathrm{~d} x+\int_{\Omega} u v \mathrm{~d} \mu=\int_{\Omega} f v \mathrm{~d} x \quad \forall v \in X_{\mu}(\Omega) .
$$

It is known that for all $\mu \in \mathcal{M}_{0}(\Omega)$ and all $f \in L^{2}(\Omega)$, there exists a unique $u_{\mu}$ solution of (2.3). (See for instance [5].)

We denote by $R_{\mu}$ the resolvent operator defined by:

$$
\begin{aligned}
R_{\mu}: L^{2}(\Omega) & \rightarrow X_{\mu}, \\
f & \mapsto u \text { solution of }(2.3) .
\end{aligned}
$$

The operator $R_{\mu}$ is linear, self-adjoint, positive, and continuous. By virtue of the Rellich Theorem, it is also compact. There exist a sequence of positive eigenvalues (going to $+\infty$ ) and a sequence of corresponding eigenfunctions (defining a Hilbert basis of $L^{2}(\Omega)$ ) that we will denote by $0<\lambda_{1}(\mu) \leq \lambda_{2}(\mu) \leq \ldots$ and $u_{1}, u_{2}, \ldots$. respectively, satisfying: for all $k, u_{k}$ is solution of the Dirichlet problem

$$
-\Delta u+u \mu=\lambda_{k}(\mu) u
$$

Since the eigenfunctions are defined up to a constant, they can be normalized so as to verify

$$
\int_{\Omega}\left|u_{k}\right|^{2} \mathrm{~d} x=1 .
$$

Note that with the notation used previously, $\lambda_{k}(0)$ is nothing else than the eigenvalue of the Laplacian $-\Delta$ in $\Omega$. In what follows, we will denote it simply by $\lambda_{k}$.

A useful tool is the variational characterization of the eigenvalues, known as the Poincaré principle or CourantFischer Formula, see [7]. Let us define the Rayleigh quotient associated to the operator $-\Delta+\mu$ by:

$$
Q R_{\mu}(u)=\frac{\int_{\Omega}|\nabla u|^{2} \mathrm{~d} x+\int_{\Omega}|u|^{2} \mathrm{~d} \mu}{\int_{\Omega}|u|^{2} \mathrm{~d} x} .
$$


Then we have

$$
\lambda_{k}(\mu)=\min _{E_{k} \subset X_{\mu}} \max _{\substack{E_{k} \\ \text { subspace of } \operatorname{dim} k}} Q E_{\mu}, u \neq 0
$$

In the formula (2.4), the minimum is taken among all the subspaces $E_{k}$ of $X_{\mu}$ of dimension $k$.

It can occur that the first eigenvalue $\lambda_{1}(\mu)$ is multiple. It is the case, for instance, if $\mu=\infty_{E}$, where $E$ is a subset of $\Omega$ such that $\Omega \backslash E$ is not connected. But this cannot happen when the total mass $\mu(\Omega)$ is finite:

Theorem 2.1. Let us assume that $\mu(\Omega)<+\infty$. Then the first eigenvalue $\lambda_{1}(\mu)$ is simple and the first eigenfunction $u_{1}$ has constant sign on $\Omega$.

This result is a consequence of a more general result proved in [3]. In the Appendix, we repeat their proof for our case.

As for the eigenfunctions' regularity, we have the following result, proved in the Appendix as well:

Lemma 2.2. Let $\lambda_{k}(\mu)$ be an eigenvalue of order $k$, and let $u$ be an eigenfunction associated to $\lambda_{k}(\mu)$. Then $u \in L^{\infty}(\Omega)$ and verifies

$$
\|u\|_{L^{\infty}(\Omega)} \leq C\|u\|_{L^{2}(\Omega)}
$$

where $C$ is a constant depending only of $N, \Omega$ and $k$.

The class $\mathcal{M}_{0}(\Omega)$ can be endowed with $\gamma$-convergence (see [10] or [2] for details). It is known that the following properties are equivalent:

$$
\begin{array}{ll}
\text { (i) } & \left(\mu_{n}\right) \gamma \text {-converges to } \mu \\
\text { (ii) } & R_{\mu_{n}} \longrightarrow R_{\mu} \text { strongly (i.e., in the operator norm) in } L^{2}(\Omega) .
\end{array}
$$

As an immediate consequence of Theorem 2.3.1 in [12], one gets that strong convergence of operators implies convergence of eigenvalues. In particular, if $\left(\mu_{n}\right) \gamma$-converge to $\mu$, then for every $k \geq 1$,

$$
\lambda_{k}\left(\mu_{n}\right) \longrightarrow \lambda_{k}(\mu)
$$

Recall a very useful tool in relaxed optimization problems: $\mathcal{M}_{0}(\Omega)$ endowed with $\gamma$-convergence is compact (see [9]).

Every measure in $\mathcal{M}_{0}(\Omega)$ can be obtained as $\gamma$-limit of a sequence in $L^{1}(\Omega)$ (see for instance [5] or [10]). Therefore, the problem (1.1) is equivalent to the following

$$
\inf _{V \in L^{1}(\Omega)}\left\{\lambda_{2}(V)-\lambda_{1}(V)+\alpha\|V\|_{L^{1}(\Omega)}\right\}
$$

The main tool in the proof of Theorem 1.1 is a result of the paper [14]. We summarize here this result, obtained by grouping Theorem 4.4 and Proposition 4.7 of [14], for the reader's convenience.

Theorem 2.3. For every $f \in L^{1}(\Omega)$, there exists a unique measure $\mu \in \mathcal{M}_{0}(\Omega)$ and a unique function $u \in X_{\mu}$ such that the couple $(u, \mu)$ satisfies the conditions:

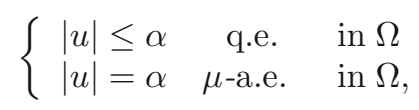

and is solution of the variational equation

$$
\int_{\Omega} \nabla u \nabla \varphi \mathrm{d} x+\int_{\Omega} u \varphi \mathrm{d} \mu=\int_{\Omega} f \varphi \mathrm{d} x, \quad \forall \varphi \in X_{\mu} \cap L^{\infty}(\Omega) .
$$


Moreover, $\mu$ verifies the following statement:

$$
\mu=\frac{1}{\alpha}\left(f ^ { + } \cdot \mathcal { L } \left\llcorner\{u=\alpha\}+f^{-} \cdot \mathcal{L}\llcorner\{u=-\alpha\}) .\right.\right.
$$

\section{Directional Derivative of an eigenvalue}

In order to obtain some necessary conditions of optimality and prove that the second eigenvalue is not degenerate at the minimum (Thm. 4.2 in the next section), we study here its directional derivative. This means that we consider the eigenvalue as a function of the measure $\mu$ defining our Schrödinger operator. We then follow the usual procedure: perturb $\mu$ linearly in a chosen direction: $\mu_{t}=\mu+t \nu$, and look for the limit of the ratio $\left[\lambda\left(\mu_{t}\right)-\lambda(\mu)\right] / t$. This approach has been developed by Kato in [13], Chapters II and VIII. We prove also at the end of this section that for $\alpha$ small enough, the solution of (1.1) is not trivial.

Let $\mu$ be a measure in $\mathcal{M}_{0}(\Omega)$. We denote by $\left\{\mu_{t}\right\}_{t>0}$ the family of measures defined by

$$
\mu_{t}=\mu+t \nu, \quad t>0
$$

where $\nu=\nu^{+}-\nu^{-}$with $\nu^{+}$and $\nu^{-}$two measures of $\mathcal{M}_{0}(\Omega)$ which verify

$$
\forall A \subset \Omega, \quad \nu^{-}(A) \leq \mu(A), \text { and } \nu^{+}(A) \leq \mu(A)+\mathcal{L}(A)
$$

It is clear that for all $t<1, \mu_{t} \in \mathcal{M}_{0}(\Omega)$ and that the norms $\|\cdot\|_{X_{\mu}},\|\cdot\|_{X_{\mu_{t}}}$ are equivalent with the following inequalities:

$$
(1-t)\|\cdot\|_{X_{\mu}} \leq\|\cdot\|_{X_{\mu_{t}}} \leq\left(1+t\left(1+\lambda_{1}^{-1}\right)\right)\|\cdot\|_{X_{\mu}}
$$

The family of operators $\left\{R_{\mu_{t}}\right\}_{t>0}$ is continuous at $t=0$. More precisely, we have:

Theorem 3.1. Let $\left\{\mu_{t}\right\}_{t>0}$ be the family of measures defined by (3.1) and (3.2). Then for $t<\frac{1}{2}$, there exists a constant $C$ depending only on $\Omega$ such that

$$
\left\|R_{\mu_{t}}(f)-R_{\mu}(f)\right\|_{X_{\mu}} \leq C t\|f\|_{L^{2}(\Omega)}
$$

Proof. Let us note in the sequel $u_{t}=R_{\mu_{t}}(f)$ and $u=R_{\mu}(f)$. Recall the variational equation verified by $u_{t}$ :

$$
\forall \varphi \in X_{\mu}, \quad \int_{\Omega} \nabla u_{t} \nabla \varphi \mathrm{d} x+\int_{\Omega} u_{t} \varphi \mathrm{d} \mu+t \int_{\Omega} u_{t} \varphi \mathrm{d} \mu=\int_{\Omega} f \varphi \mathrm{d} x .
$$

By taking $u_{t}$ as test function in (3.4) and thanks to the Poincaré inequality, we obtain

$$
\left\|u_{t}\right\|_{L^{2}(\Omega)} \leq \frac{\|f\|_{L^{2}(\Omega)}}{\lambda_{1}}
$$

Consider now the variational equation solved by $u_{t}-u$ :

$$
\forall \varphi \in X_{\mu}, \quad \int_{\Omega} \nabla\left(u_{t}-u\right) \nabla \varphi \mathrm{d} x+\int_{\Omega}\left(u_{t}-u\right) \varphi \mathrm{d} \mu=-t \int_{\Omega} u_{t}\left(u_{t}-u\right) \varphi \mathrm{d} \nu
$$

By taking $u_{t}-u$ as test function in (3.5) and using the Cauchy-Schwartz inequality, we get

$$
\left\|u_{t}-u\right\|_{X_{\mu}}^{2} \leq t \frac{\|f\|_{L^{2}(\Omega)}}{\lambda_{1}} \int_{\Omega}\left|u_{t}-u\right|^{2} \mathrm{~d}\left(\nu^{+}+\nu^{-}\right) .
$$


Thanks to the condition (3.2), for all $v \in X_{\mu}$,

$$
\int_{\Omega}|v|^{2} \mathrm{~d}\left(\nu^{+}+\nu^{-}\right) \leq\left(2+\frac{1}{\lambda_{1}}\right)\|v\|_{X_{\mu}} .
$$

(3.6) and (3.7) together end the proof.

As a consequence of this theorem, we have the following corollary.

Corollary 3.2. Let $\left\{f_{t}\right\}_{t>0}$ be a family of functions such that $\left(f_{t}\right)$ converges weakly to $f$ in $L^{2}(\Omega)$. Then

$$
\lim _{t \rightarrow 0}\left\|R_{\mu_{t}}\left(f_{t}\right)-R_{\mu}(f)\right\|_{X_{\mu}}=0
$$

Proof. First of all, we note that

$$
\left\|R_{\mu_{t}}\left(f_{t}\right)-R_{\mu}(f)\right\|_{X_{\mu}} \leq\left\|R_{\mu_{t}}\left(f_{t}\right)-R_{\mu}\left(f_{t}\right)\right\|_{X_{\mu}}+\left\|R_{\mu}\left(f_{t}\right)-R_{\mu}(f)\right\|_{X_{\mu}}
$$

Since the family $\left(f_{t}\right)$ is uniformly bounded in $L^{2}(\Omega)$ norm, by Theorem 3.1, the first term at the right-hand side of the above inequality goes to zero at $t=0$. For proving that the second term goes to zero as well, we follow a classical method. Let us note $u_{t}=R_{\mu}\left(f_{t}\right)$; then $u_{t}$ solves:

$$
\forall \varphi \in X_{\mu}, \quad \int_{\Omega} \nabla u_{t} \nabla \varphi \mathrm{d} x+\int_{\Omega} u_{t} \varphi \mathrm{d} \mu=\int_{\Omega} f_{t} \varphi \mathrm{d} x
$$

By taking $u_{t}$ as test function in (3.8) and thanks to the Poincaré inequality, we obtain

$$
\left\|u_{t}\right\|_{X_{\mu}}^{2} \leq \frac{\|f\|_{L^{2}(\Omega)}^{2}}{\lambda_{1}}
$$

Hence, every sequence $\left(u_{t_{n}}\right)$, where $\left(t_{n}\right)$ is a positive sequence converging to zero as $n$ goes to infinity, has a subsequence converging weakly in $X_{\mu}$ to a function $u \in X_{\mu}$. By the Rellich theorem, this subsequence converges strongly to $u$ in $L^{2}(\Omega)$. Passing to the limit in (3.8), we obtain that $u=R_{\mu}(f)$. Since the limit is unique, all the sequence $\left(u_{t_{n}}\right)$ converges to $u$ and so,

$$
\lim _{t \rightarrow 0}\left\|u_{t}-u\right\|_{L^{2}(\Omega)}=0
$$

By taking $u_{t}$, once again, as test function in (3.8) and by passing to the limit, we obtain that the convergence holds in $X_{\mu}$. This ends the proof.

As an immediate consequence of Theorem 3.1 we get that $R_{\mu_{t}}$ converges to $R_{\mu}$ in the operator norm in $L^{2}(\Omega)$, and consequently, for every $k \in \mathbb{N}^{*}$ (see [12]):

$$
\lim _{t \rightarrow 0} \lambda_{k}\left(\mu_{t}\right)=\lambda_{k}(\mu)
$$

The next result deals with derivatives of eigenvalues. Note that we will not apply the general results of Kato [13], since the derivability proof comes in our case rather directly from what has been said up to now.

Theorem 3.3. Let $\lambda_{k}(\mu)$ an eigenvalue of multiplicity $m$ such that $\lambda_{k}(\mu)=\ldots=\lambda_{k+m-1}(\mu)$. Let $\left(t_{n}\right)$ be a positive sequence converging to zero as $n$ goes to $+\infty$. There exists $\left\{u_{k} ; \ldots ; u_{k+m-1}\right\}$ an orthonormal family 
of eigenfunctions associated to $\lambda_{k}(\mu)$, and a subsequence of $\left(t_{n}\right)$ (denoted with the same index) such that for all $(i ; j) \in[k ; k+m-1]^{2}, i \neq j:$

$$
\begin{array}{ll}
\text { (i) } & \frac{\lambda_{i}\left(\mu_{t_{n}}\right)-\lambda_{i}(\mu)}{t_{n}} \longrightarrow \int_{\Omega}\left|u_{i}\right|^{2} \mathrm{~d} \nu, \\
\text { (ii) } & \int_{\Omega} u_{i} u_{j} \mathrm{~d} \nu=0 .
\end{array}
$$

To prove Theorem 3.3, we need the following technical lemma:

Lemma 3.4. Let $u_{p, t}$ be an eigenvector associated to an eigenvalue $\lambda_{p}\left(\mu_{t}\right)$, and $u_{q}$ an eigenvector associated to $\lambda_{q}(\mu)$. Then for all $t>0$,

$$
\frac{\lambda_{p}\left(\mu_{t}\right)-\lambda_{q}(\mu)}{t} \int_{\Omega} u_{p, t} u_{q} \mathrm{~d} x=\int_{\Omega} u_{p, t} u_{q} \mathrm{~d} \nu .
$$

Proof. Since $X_{\mu}=X_{\mu_{t}}$, we can take $u_{q}$ as test function in the equation solved by $u_{p, t}$ and $u_{p, t}$ as test function in the equation solved by $u_{q}$. We obtain

$$
\begin{gathered}
\int_{\Omega} \nabla u_{p, t} \nabla u_{q} \mathrm{~d} x+\int_{\Omega} u_{p, t} u_{q} \mathrm{~d} \mu_{t}=\lambda_{p}\left(\mu_{t}\right) \int_{\Omega} u_{p, t} u_{q} \mathrm{~d} x, \quad \text { and } \\
\int_{\Omega} \nabla u_{q} \nabla u_{p, t} \mathrm{~d} x+\int_{\Omega} u_{q} u_{p, t} \mathrm{~d} \mu=\lambda_{p}(\mu) \int_{\Omega} u_{q} u_{p, t} \mathrm{~d} x .
\end{gathered}
$$

By taking the difference of both equalities, we obtain the result.

Proof of Theorem 3.3. Let us denote by $\left\{u_{k, t} ; \ldots ; u_{k+m-1, t}\right\}$ an orthonormal (for the $L^{2}(\Omega)$ scalar product) family of eigenfunctions associated to eigenvalues $\left\{\lambda_{k}\left(\mu_{t}\right) ; \ldots ; \lambda_{k+m-1}\left(\mu_{t}\right)\right\}$. Up to a subsequence, for all $i \in[k ; k+m-1], u_{i, t_{n}}$ converges weakly in $L^{2}(\Omega)$ to a function $u_{i}$. By Corollary 3.2 , we know that $R_{\mu_{t_{n}}}\left(u_{i, t_{n}}\right)$ converges in $X_{\mu}$ strongly to $R_{\mu}\left(u_{i}\right)$. But since

$$
R_{\mu_{t_{n}}}\left(u_{i, t_{n}}\right)=\frac{1}{\lambda_{i}\left(\mu_{t_{n}}\right)} u_{i, t_{n}}
$$

the sequence $u_{i, t_{n}}$ converges to $u_{i}$ strongly in $X_{\mu}$. So, $u_{i}$ is a normalized eigenfunction associated to $\lambda_{i}(\mu)$ and $\left\{u_{k} ; \ldots ; u_{k+m-1}\right\}$ is an orthogonal family in $L^{2}(\Omega)$. By $(3.2), u_{i, t_{n}}$ converges to $u_{i}$ in $L_{\nu^{+}}^{2}(\Omega)$ and in $L_{\nu^{-}}^{2}(\Omega)$. By virtue of Lemma 3.4,

$$
\frac{\lambda_{i}\left(\mu_{t_{n}}\right)-\lambda_{i}(\mu)}{t_{n}} \int_{\Omega} u_{i, t_{n}} u_{i} \mathrm{~d} x=\int_{\Omega} u_{i, t_{n}} u_{i} \mathrm{~d} \nu .
$$

When passing to the limit in this equality, we get $(i)$. Now, with $i \neq j$,

$$
\frac{\lambda_{i}\left(\mu_{t_{n}}\right)-\lambda_{j}(\mu)}{t_{n}} \int_{\Omega} u_{i, t_{n}} u_{j} \mathrm{~d} x=\int_{\Omega} u_{i, t_{n}} u_{j} \mathrm{~d} \nu .
$$

But as $\lambda_{j}(\mu)=\lambda_{i}(\mu)$, when passing to the limit, we obtain $(i i)$.

Theorem 3.3 applied at $t=0$ allows to prove that the trivial solution is not a solution of problem (1.1).

Proposition 3.5. For $\alpha$ small enough, the solution of (1.1) is not trivial. 
Proof. Applying Theorem 3.3 with $\mu \equiv 0$, we obtain that for every measure $\nu$ absolutely continuous with respect to the Lebesgue measure,

$$
\lim _{t \rightarrow 0} \frac{1}{t}\left[\left(\lambda_{2}\left(\mu_{t}\right)-\lambda_{1}\left(\mu_{t}\right)+t \alpha \nu(\Omega)\right)-\left(\lambda_{2}(0)-\lambda_{1}(0)\right)\right]=\int_{\Omega}\left(u_{2}^{2}-u_{1}^{2}+\alpha\right) \mathrm{d} \nu .
$$

We assume that $\mu \equiv 0$ is optimal. This means that the limit above is nonnegative for every admissible measure $\nu$. Then $u_{2}^{2}-u_{1}^{2}+\alpha \geq 0$ a.e. in $\Omega$. But this is impossible for $\alpha$ small enough. Otherwise, we would obtain that $u_{2}^{2}=u_{1}^{2}$ a.e. in $\Omega$, and so $\left|\nabla u_{2}\right|^{2}=\left|\nabla u_{1}\right|^{2}$ a.e. in $\Omega$; consequently $\lambda_{1}(0)=\lambda_{2}(0)$, which contradicts connectedness of $\Omega$.

\section{THE RESULT}

We first deal with the problem of existence of solutions.

Theorem 4.1. The problem (1.1) has at least one solution.

Proof. Let $\left(\mu_{n}\right)$ be a minimizing sequence for the problem (1.1). Up to a subsequence, we may assume that $\left(\mu_{n}\right) \gamma$-converges to a measure $\mu$ which belongs to $\mathcal{M}_{0}(\Omega)$. Since $\lambda_{2}(\mu)$ and $\lambda_{1}(\mu)$ are continuous and $\mu(\Omega)$ is lower semi-continuous under $\gamma$-convergence (see [6]), the measure $\mu$ is a solution of (1.1).

The next theorem gives some necessary conditions of optimality for the solution of the optimization problem (1.1), as well as the simplicity of the optimal eigenvalue.

Theorem 4.2. Let $\mu$ be a solution to the problem (1.1) with $\alpha<|\Omega|^{-1}$. Then:

(a) $\quad \lambda_{1}(\mu)$ and $\lambda_{2}(\mu)$ are non degenerate;

(b) for $u_{1}, u_{2}$ two normalized eigenvectors associated respectively

to $\lambda_{1}(\mu)$ and $\lambda_{2}(\mu)$, one has

(i) $\quad u_{2}^{2}-u_{1}^{2}+\alpha \geq 0$ a.e. $n \Omega$,

(ii) $\quad u_{2}^{2}-u_{1}^{2}+\alpha=0 \quad \mu$-a.e. in $\Omega$.

Proof. $\lambda_{1}(\mu)$ is non degenerate thanks to Theorem 2.1. Let us now prove $(b)$.

According to Theorem 3.3, there exist: an orthonormal family $\left\{u_{1} ; u_{2} ; \ldots ; u_{m}\right\}$ of eigenfunctions where $u_{1}$ is associated to $\lambda_{1}(\mu)$ and $\left\{u_{2} ; \ldots ; u_{m}\right\}$ are associated to $\lambda_{2}(\mu)$ and a positive sequence $\left(t_{n}\right)$ converging to zero such that the conclusion of the theorem are valid for $k=1$ and $k=2$. Since $\mu$ is optimal, for all $t>0$,

$$
\frac{\lambda_{2}\left(\mu_{t}\right)-\lambda_{2}(\mu)}{t}-\frac{\lambda_{1}\left(\mu_{t}\right)-\lambda_{1}(\mu)}{t}-\alpha \nu(\Omega) \geq 0 .
$$

But, for every $k \geq 2, \lambda_{k}\left(\mu_{t}\right) \geq \lambda_{2}\left(\mu_{t}\right)$ and $\lambda_{k}(\mu)=\lambda_{2}(\mu)$, then for all $t>0$ :

$$
\frac{\lambda_{k}\left(\mu_{t}\right)-\lambda_{k}(\mu)}{t}-\frac{\lambda_{1}\left(\mu_{t}\right)-\lambda_{1}(\mu)}{t}-\alpha \nu(\Omega) \geq 0 .
$$

Passing to the limit in (4.1), we obtain

$$
\int_{\Omega}\left(\left|u_{k}\right|^{2}-\left|u_{1}\right|^{2}+\alpha\right) \mathrm{d} \nu \geq 0 .
$$

Suppose that $u$ is any normalized eigenfunction associated to $\lambda_{2}(\mu)$. We can write

$$
u=\sum_{i=2}^{m} \alpha_{i} e^{(i)}, \text { with } \sum_{i=2}^{m}\left|\alpha_{i}\right|^{2}=1 .
$$


Because of $(i i)$ of Theorem 3.3

$$
\int_{\Omega}\left(|u|^{2}-\left|u_{1}\right|^{2}+\alpha\right) \mathrm{d} \nu=\int_{\Omega} \sum_{i=2}^{m}\left|\alpha_{i}\right|^{2}\left(\left|u_{i}\right|^{2}-\left|u_{1}\right|^{2}+\alpha\right) \mathrm{d} \nu
$$

Finally, using (4.2), we can conclude that for any normalized eigenfunction $u$ associated to $\lambda_{2}(\mu)$ and for every measure $\nu$ respecting conditions (3.2),

$$
\int_{\Omega}\left(|u|^{2}-\left|u_{1}\right|^{2}+\alpha\right) \mathrm{d} \nu \geq 0
$$

The inequality (4.3) is valid for any measure $\nu$ of the form $\nu=\varphi \cdot \mathcal{L}$ where $\varphi$ is a nonnegative function belonging to $L^{\infty}(\Omega)$ and bounded by one. Since $\varphi$ are arbitrary, it gives that $|u|^{2}-\left|u_{1}\right|^{2}+\alpha \geq 0$ a.e. in $\Omega$ and also q.e. in $\Omega$ because the functions $u_{1}$ and $u_{2}$ are quasi-continuous. Let us now consider $\nu=-\mu$ in (4.3): we obtain that $|u|^{2}-\left|u_{1}\right|^{2}+\alpha=0 \mu$-a.e. in $\Omega$.

Let us now prove $(a)$. Assume that $\lambda_{2}(\mu)$ is degenerate, then there exist $u_{2}$ and $u_{3}$ two orthonormal eigenfunctions associated to $\lambda_{2}(\mu)$. Since $\alpha<|\Omega|^{-1}$, there exists $\delta>0$ such that $\left\{u_{1}^{2}>\alpha+\delta\right\}$ is not a polar set for the Lebesgue measure. Let $x$ be a Lebesgue point of $u_{1}, u_{2}, u_{3}$ and belonging to $\left\{u_{1}^{2}>\alpha+\delta\right\}$. Let us first note that $u_{2}(x)$ and $u_{3}(x)$ are not equal to zero since from the optimality condition $(b-i)$, we have

$$
\oint u_{2}^{2}(y) \mathrm{d} y \geq \oint\left(u_{1}^{2}(y)-\alpha\right) \mathrm{d} y
$$

so, passing to the limit in (4.4), we get $u_{2}^{2}(x) \geq \delta$. (We prove in the same way that $u_{3}(x) \neq 0$.) Consider $u$ defined by $u=\beta u_{2}+\gamma u_{3}$ where $\beta^{2}+\gamma^{2}=1$ and $\alpha u_{2}(x)+\gamma u_{3}(x)=0$ (which is possible since $u_{2}(x), u_{3}(x)$ are not equal to zero). The function $u$ is a normalized eigenfunction associated to $\lambda_{2}(\mu)$. By the optimality condition $(b-i)$ and the fact that $x$ is a Lebesgue point of $u_{1}$ in $\left\{u_{1}^{2}(x) \geq \alpha+\delta\right\}$, we get

$$
\liminf _{\varepsilon \rightarrow 0} \oint|u(y)|^{2} \mathrm{~d} y \geq \delta
$$

On the other hand,

$$
\oint|u(y)-u(x)|^{2} \mathrm{~d} y \leq 2\left(\beta^{2} \oint|u(y)-u(x)|^{2} \mathrm{~d} y+\gamma^{2} \oint|u(y)-u(x)|^{2} \mathrm{~d} y\right) .
$$

Since $u(x)=0$, it implies that

$$
\lim _{\varepsilon \rightarrow 0} \oint|u(y)|^{2} \mathrm{~d} y=0
$$

which contradicts (4.5) and ends the proof.

We now give and prove the main result of this paper.

Theorem 4.3. Let $\mu$ be a solution of the problem (1.1) with $\alpha<|\Omega|^{-1}$. Then $\mu$ is absolutely continuous with respect to the Lebesgue measure and verifies

$$
\mu=\frac{2}{\alpha}\left[\lambda_{1}(\mu) u_{1}^{2}-\lambda_{2}(\mu) u_{2}^{2}+\left|\nabla u_{2}\right|^{2}-\left|\nabla u_{1}\right|^{2}\right] \cdot \mathcal{L}_{\left\{u_{1}^{2}-u_{2}^{2}=\alpha\right\}}
$$

where $u_{1}$ and $u_{2}$ are two normalized eigenfunctions associated respectively to $\lambda_{1}(\mu)$ and $\lambda_{2}(\mu)$. 
Proof. Let $w:=u_{1}^{2}-u_{2}^{2}$, then by the Lemma 2.2, $w \in X_{\mu} \cap L^{\infty}(\Omega)$. A simple computation shows that $w$ is solution of

$$
\int_{\Omega} \nabla w \nabla \varphi \mathrm{d} x+\int_{\Omega} w \varphi \mathrm{d} \mu=\int_{\Omega} g \varphi \mathrm{d} x, \forall \varphi \in X_{\mu} \cap L^{\infty}(\Omega),
$$

where $g=2\left(\lambda_{1}(\mu) u_{1}^{2}-\lambda_{2}(\mu) u_{2}^{2}+\left|\nabla u_{2}\right|^{2}-\left|\nabla u_{1}\right|^{2}\right)$. It is clear that $g$ belongs to $L^{1}(\Omega)$. Let $w^{+}$be the positive part of $w$. By taking $w^{+} \varphi$ as test function in (4.6) we obtain that $w^{+}$, which belongs to $X_{\mu} \cap L^{\infty}(\Omega)$, is solution of

$$
\int_{\Omega} \nabla\left(w^{+}\right)^{2} \nabla \varphi \mathrm{d} x+2 \int_{\Omega}\left(w^{+}\right)^{2} \varphi \mathrm{d} \mu=\int_{\Omega} 2\left(g w^{+}-\left|\nabla w^{+}\right|^{2}\right) \varphi \mathrm{d} x, \quad \forall \varphi \in X_{\mu} \cap L^{\infty}(\Omega) .
$$

From Theorem 4.2, it comes that $\left(w^{+}\right)^{2}$ satisfies $\left(w^{+}\right)^{2} \leq \alpha^{2}$ q.e. in $\Omega$ and $\left(w^{+}\right)^{2}=\alpha^{2} \mu$-a.e. in $\Omega$. Then, applying Theorem 2.3, we obtain

$$
\mu=\frac{1}{\alpha^{2}}\left(g w^{+}-\left|\nabla w^{+}\right|^{2}\right)^{+} \chi_{K}
$$

where $K=\left\{w^{+}=\alpha\right\}$. But since $\left|\nabla w^{+}\right|=0$ a.e. in $K$, we obtain that

$$
\mu=\frac{1}{\alpha} g \chi_{K}
$$

This ends the proof.

\section{Appendix}

In this section, we give the proofs of Theorem 2.1 and Lemma 2.2.

Theorem 5.1. Let us assume that $\mu(\Omega)<+\infty$. Then the first eigenvalue $\lambda_{1}(\mu)$ is simple and the first eigenfunction associated to $\lambda_{1}(\mu)$ has constant sign on $\Omega$.

In order to prove this result, we need some results related to capacity of Sobolev functions. As in this paper, we do not want to enter too much into the details, we refer to $[2,15]$ for a very complete description of the properties of Sobolev function and to [4] for the definition of a quasi-connected open set.

Proof. Let us denote by $u$ a first eigenfunction. It is known that one has

$$
Q R_{\mu}(u) \geq \min \left\{Q R_{\mu}\left(u^{+}\right), Q R_{\mu}\left(u^{-}\right)\right\} .
$$

Then either $u^{+}$or $u^{-}$is an eigenfunction, and by linearity the other one is an eigenfunction too. So we can suppose that $u$ is non negative in $\Omega$.

Let us note $F:=\{u=0\}$. To prove that $\lambda_{1}(\mu)$ is simple, it is sufficient to prove that $\operatorname{Cap}(F)=0$. Indeed, in this case, any other first eigenvector orthogonal to $u$ is equal to zero. Let $K$ be a smooth connected compact set included in $\Omega$. Consider the $\mu$-capacity of $F \cap K$ in $\Omega$ defined by

$$
\operatorname{Cap}_{\mu}(F \cap K)=\inf _{\substack{v \in X_{\mu} \\ v \geq 1 \text { in } F \cap K}}\left\{\int_{\Omega}|\nabla v|^{2} \mathrm{~d} x+\int_{\Omega}|v|^{2} \mathrm{~d} \mu\right\} .
$$

Since $K \subset \Omega$ and $\mu(\Omega)$ is bounded, we have $\operatorname{Cap}_{\mu}(F \cap K)<+\infty$. So, there exists $w$ which realizes the infimum of (5.1) (see [8] for details about $\mu$-capacity). For every $\varphi \in H_{0}^{1}(\Omega \backslash F) \cap L_{\mu}^{2}(\Omega)$ the Euler equation yields:

$$
\int_{\Omega} \nabla w \nabla \varphi \mathrm{d} x+\int_{\Omega} w \varphi \mathrm{d} \mu=0
$$


Since $u \in H_{0}^{1}(\Omega \backslash F) \cap L_{\mu}^{2}(\Omega)$, equation (5.2) is verified with $u$ as test function. Then we obtain

$$
\int_{\Omega} u w \mathrm{~d} x=0
$$

Hence $w=0$ q.e. in $\{u>0\}$. Consider the quasi-open set $\left\{w>\frac{1}{2}\right\}$. Up to a set of zero capacity, $K$ is included in $\{u>0\} \cup\left\{w>\frac{1}{2}\right\}$ with $\{u>0\} \cap\left\{w>\frac{1}{2}\right\}=\emptyset$. But since $K$ is connected, $\operatorname{Cap}(F \cap K)=0$. And as it is realized for every smooth connected compact set $K \in \Omega$, we have $\operatorname{Cap}(F)=0$.

Lemma 5.2. Let $\lambda_{k}(\mu)$ be an eigenvalue of order $k$, and let $u$ be an eigenfunction associated to $\lambda_{k}(\mu)$. Then $u \in L^{\infty}(\Omega)$ and verifies

$$
\|u\|_{L^{\infty}(\Omega)} \leq C\|u\|_{L^{2}(\Omega)} .
$$

Here, $C$ is a constant depending only on $N, \Omega$ and $k$.

Proof. Let us note $\lambda=\lambda_{k}(\mu)$. Let $w_{+}$and $w_{-}$be respectively the weak solutions of

$$
-\Delta w_{+}+w_{+} \mu=\lambda u^{+} \quad \text { and } \quad-\Delta w_{-}+w_{-} \mu=\lambda u^{-} \quad \text { in } \Omega,
$$

where $u^{+}=\max (u, 0)$ and $u^{-}=\min (u, 0)$. By the maximum principle, $-w_{-} \leq u \leq w_{+}$a.e. in $\Omega$. Let $w$ be the weak solution of

$$
-\Delta w=\lambda|u| \text { in } \Omega .
$$

Then, also by the maximum principle, $w_{+} \leq w$ and $w_{-} \leq w$ a.e. in $\Omega$. Hence $|u| \leq w$ in $\Omega$. Moreover, the regularity of the weak solution implies that $w \in H^{2}(\Omega)$ with:

$$
\|w\|_{H^{2}(\Omega)} \leq \lambda C\|u\|_{L^{2}(\Omega)},
$$

where $C=C(N, \Omega)$. Let us define the sequence $\left(w_{n}\right)_{n \in \mathbb{N}}$ by $w_{1}=w$ and for every $n \geq 2$ :

$$
w_{n+1} \in H_{0}^{1}(\Omega) \text {, and }-\Delta w_{n+1}=\lambda w_{n} \quad \text { in } \Omega .
$$

By induction, for every $n \geq 1$,

$$
w_{n+1} \geq w_{n}, \quad \text { and, } \quad\left\|w_{n}\right\|_{H^{2 n}(\Omega)} \leq \lambda C^{n}\|u\|_{L^{2}(\Omega)} .
$$

Hence we have $|u| \leq w_{n}$ and for every $n>\frac{N}{4}, w_{n} \in L^{\infty}(\Omega)$ with $\left\|w_{n}\right\|_{L^{\infty}(\Omega)} \leq C\|u\|_{L^{2}(\Omega)}$ where $C=C(N, \Omega, k)$. This ends the proof.

\section{REFERENCES}

[1] M.S. Ashbaugh, E.M. Harrell and R. Svirsky, On minimal and maximal eigenvalue gaps and their causes. Pacific J. Math. 147 (1991) 1-24.

[2] D. Bucur and G. Buttazzo, Variational Methods in Shape Optimization Problems, Progress in Nonlinear Differential Equations and Their Applications 65. Birkhäuser, Basel, Boston (2005).

[3] D. Bucur and T. Chatelain, Strict monotonicity of the second eigenvalue of the Laplace operator on relaxed domain. Bull. Appl. Comp. Math. 1510-1566 (1998) 115-122.

[4] D. Bucur and A. Henrot, Minimization of the third eigenvalue of the Dirichlet Laplacian. Proc. Roy. Soc. London 456 (2000) 985-996.

[5] G. Buttazzo and G. Dal Maso, Shape optimization for Dirichlet problems: relaxed formulation and optimality conditions. Appl. Math. Optim. 23 (1991) 17-49. 
[6] G. Buttazzo, N. Varchon and H. Zoubairi, Optimal measures for elliptic problems. Annali Mat. Pur. Appl. 185 (2006) $207-221$.

[7] R. Courant and D. Hilbert, Methods of Mathematical Physics. Interscience Publishers (1953).

[8] G. Dal Maso, Г-convergence and $\mu$-capacities. Ann. Scuola Norm. Sup. Pisa Cl. Sci. (4) 14 (1987) $423-464$.

[9] G. Dal Maso, An introduction to $\Gamma$-convergence. Birkhäuser, Boston (1993).

[10] G. Dal Maso and U. Mosco, Wiener's criterion and Г-convergence. Appl. Math. Optim. 15 (1987) 15-63.

[11] L.C. Evans and R.F. Gariepy, Measure theory and fine properties of functions, Studies in Advanced Mathematics. CRC Press, Boca Raton (1992).

[12] A. Henrot, Extremum Problems for Eigenvalues of Elliptic Operators. Birkhäuser Verlag, Basel, Boston, Berlin (2006).

[13] T. Kato, Perturbation Theory for Linear Operators. Springer-Verlag (1980).

[14] N. Varchon, Optimal measures for nonlinear cost functionals. Appl. Mat. Opt. 54 (2006) $205-221$.

[15] W.P. Ziemer, Weakly Differentiable Functions. Springer-Verlag, Berlin (1989). 